Article

\title{
Establishment of the Volatile Signature of Wine-Based Aromatic Vinegars Subjected to Maceration
}

\author{
Rosa Perestrelo ${ }^{1}$, Catarina L. Silva ${ }^{1}$, Pedro Silva ${ }^{1}$ (i) and José S. Câmara ${ }^{1,2, *}$ \\ 1 CQM-Centro de Química da Madeira, Universidade da Madeira, Campus da Penteada, \\ 9020-105 Funchal, Portugal; rmp@uma.pt (R.P.); cgsluis@uma.pt (C.L.S.); pedro_dasilva@hotmail.com (P.S.) \\ 2 Departamento de Química, Faculdade de Ciências Exatas e Engenharia, Universidade da Madeira, \\ Campus da Penteada, 9020-105 Funchal, Portugal \\ * Correspondence: jsc@uma.pt; Tel.: +351-291705112
}

Received: 17 January 2018; Accepted: 18 February 2018; Published: 23 February 2018

\begin{abstract}
The flavoring of vinegars with aromatic fruits and medicinal herbs is a practice with increasing trend mostly in countries with oenological tradition, resulting in a product of improved quality and consumer attractiveness. This study was directed towards the evaluation of the impact of the maceration process on the volatile signature of wine-based aromatic vinegars (WBAVs). The evaluation was performed using solid phase microextraction (SPME) combined with gas chromatography combined with mass spectrometry (GC-MS). Experimental parameters influencing headspace solid (HS)-SPME extraction efficiency, were optimized using an univariate experimental design. The best results were achieved using a polydimethylsiloxane (PDMS) fiber, $10 \mathrm{~mL}$ of vinegar sample, at $50^{\circ} \mathrm{C}$ for $30 \mathrm{~min}$ of extraction. This way One hundred and three volatile organic compounds (VOCs), belonging to different chemical families including ethyl esters (37), higher alcohols (20), fatty acids (10), terpenoids (23), carbonyl compounds (six), lactones (five) and volatile phenols (two), were identified in wine vinegar (control) and WBAV. As far as we know, 34 of these VOCs are reported for the first time in macerated vinegars. Higher alcohols and lactones are the major chemical families in WBAV macerated with apple, whereas terpenoids are predominant in WBAV macerated with banana. The obtained data represent a suitable tool to guarantee the authenticity and genuineness of WBAV, as well as to promote the production of WBAV with improved sensorial and organoleptic properties. To the best of our knowledge, there are no reported studies dealing with the volatile signature of WBAV enriched with banana, passion fruit, apple and pennyroyal.
\end{abstract}

Keywords: vinegars; volatile signature; HS-SPME/GC-MS; PCA; authenticity

\section{Introduction}

Vinegar is a traditional food product with a high reputation throughout the world, used not only as a condiment, but also as preserving agent for a wide range of foods [1]. It is produced from raw materials containing mainly carbohydrates in two-stage fermentation processes, where the first one involves ethanol formation by yeasts (usually Saccharomyces species) through the conversion of fermentable sugars (alcoholic fermentation) and subsequently the oxidation of ethanol to acetic acid (acetification). From a technological point of view, there are mainly two defined vinegar making methods (Figure 1): (i) the traditional method, in which the culture of acetic acid bacteria is placed on the surface of a barrel in a direct contact with oxygen; and (ii) the industrial method, a quick process involving a submerged culture where the oxygenation has been greatly improved [2-4].

The market is turning towards the diversity of food and ingredients that prevent several diseases, such as those showing antibacterial, anti-inflammatory, blood glucose control, lipid metabolism 
regulation, weight loss, anticancer and cardiovascular disease prevention activity [2,5-7]. Taking into account these reasons, the agri-food market is focused on the development of novel products based on traditional processes with higher nutritional and organoleptic properties. Following the research line of development of products with increased value from a nutritional point of view, a new sherry vinegar-derived product enriched with dietary fiber has been developed by Marrufo-Curtido et al. [8]. Venturi et al. [9] developed a phenol-enriched refined olive oil with its own phenolic compounds extracted from wastewater produced during physical processing in order to improve the nutraceutical value, as well as the antioxidant capacity of olive oil. On the other hand, marine sources (e.g., macroalgae, microalgae), food by-products and plant-derived natural products can be used to extract bioactive compounds, such as polyunsaturated fatty acids (PUFAs) and phenolics, among others, that are widely recognized as important nutritional components that may help prevent various cardiac disorders [10-13].

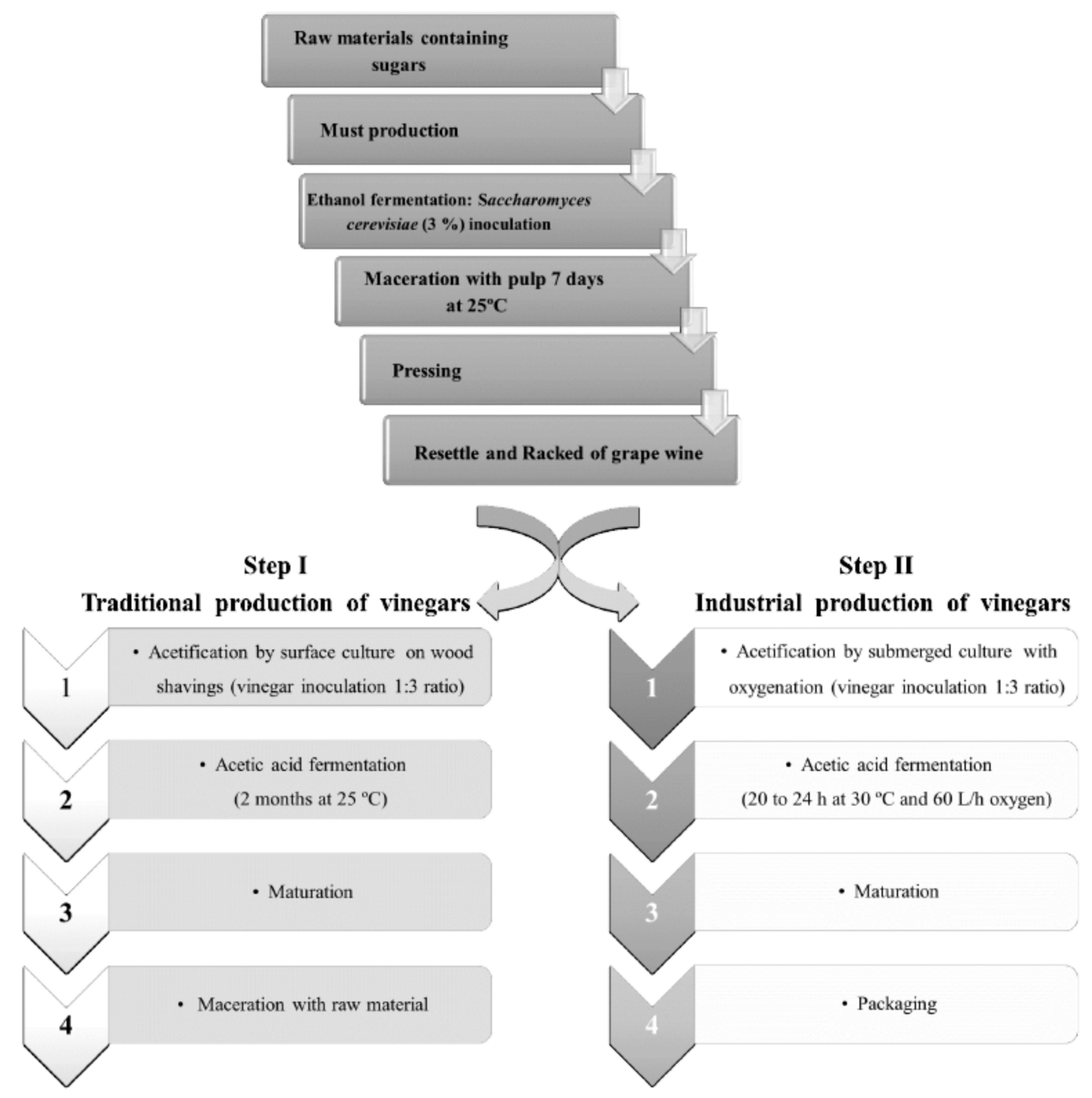

Figure 1. Flowchart showing the general production methods for vinegar [2-4].

Recently, new products associated with several fruits have arisen, such as vinegars macerated with fruits, fruit juice with added vinegar and fruit vinegars, which improve the organoleptic and health-promoting characteristics [5-7,14-17]. Its quality and acceptance by consumers depends on several parameters, being aroma one of the most important. In wine-based vinegars and derived products, aroma results from several hundreds of volatile organic compounds (VOCs) belonging 
to different chemical families (e.g., mono- and sesquiterpenes, esters, higher alcohols, carbonyl and sulphur compounds) encompassing a wide range of volatilities and polarities. These VOCs may come from the raw materials (e.g., red wines, fruits, cider, malted barley, honey, among others), and/or may be formed during production and storage processes [1,4,6,14-18]. VOCs are present, to a large extent, in fruits and aromatic/medicinal herbs influencing positively the final quality of vinegars by the addition of new compounds derived from them. In addition, fruits are rich in vitamins, minerals, phytochemicals, and contain potentially bioactive compounds including polyphenols (e.g., flavonoids and non-flavonoids) which have been shown to prevent oxidative processes. These compounds confer to fruits a significant antioxidant capacity related to numerous healthy properties [2,5-7].

Due to the diversity of commercially available vinegars and vinegar-based products and its increasing demand trend, the development of reliable and high throughput analytical methodologies able to establish criteria for determining its quality and origin are crucial to achieve this purpose [19]. Several extraction techniques, such as steam distillation [20], liquid-liquid extraction [21], stir bar sorptive extraction [5], solid-phase extraction [22], and solid-phase microextraction (SPME) [23] have been applied to isolate the volatiles from vinegars and vinegar-derived products. Compared to other extraction processes SPME offers several advantages such as the fact it eliminates the use of extraction solvent and allows the extraction and the concentration steps to be performed simultaneously [23]. The vinegar volatile signature is usually established using chromatographic techniques, in particular gas chromatography combined with mass spectrometry (GC-MS) [24].

The characterization and discrimination of vinegars have been widely investigated through the integration of chromatographic data with chemometric tools, such as principal component analysis (PCA) and hierarchical cluster analysis (HCA). PCA and HCA are among the most used chemometric tools to view, analyze and explore the large information provided by the analytical instrumentation. These two pattern recognition tools complement one another and have been widely applied to solve classification problems $[25,26]$.

Following the research line of novel aromatic vinegars, with improved nutritional and sensorial properties, the aim of the current work was the establishment of he volatile signature of a wine vinegar (control) and wine-based aromatic vinegars (WBAVs) macerated with different fruits (e.g., banana, passion fruit and apple) and a medicinal herb (e.g., pennyroyal) using headspace solid-phase microextraction (HS-SPME) combined with gas chromatography-mass spectrometry (GC-MS). In addition, chemometric tools, namely PCA and HCA, were applied to obtain deep insights into variations on the volatile signature of target vinegars, and to identify the VOCs responsible for the discrimination among vinegars. Our study's findings could provide to "home-made" producers new opportunities to promote the production of wine vinegars with enhanced levels of odoriferous VOCs, to guarantee the vinegars typicality and to expand the oenological market. To the best of authors' knowledge, there has not been any report on the volatile signatures of WBAV macerated with banana, passion fruit, apple and pennyroyal.

\section{Results and Discussion}

\subsection{HS-SPME Optimization}

To develop a suitable and powerful HS-SPME/GC-MS method in order to establish the volatile signature of wine vinegar (control) and WBAV (banana, passion fruit, apple and pennyroyal), the most important experimental parameters influencing the HS-SPME extraction efficiency, including fiber coating, extraction time, extraction temperature, sample volume, ionic strength and desorption time, were optimized using an univariate experimental design. The optimal extraction conditions were chosen based on total GC peak area, number of isolated and identified VOCs and precision (expressed as relative standard deviation, $\%$ RSD). 


\subsubsection{Fiber Coating}

The selection of the most suitable SPME fiber depends on the composition of the sample material under study [27]. Five fiber coatings, including carboxen/polydimethylsiloxane (CAR/PDMS, $75 \mu \mathrm{m}$ ), divinylbenzene/carboxen on polydimethylsiloxane (DBV/CAR/PDMS; StableFlex, 50/30 $\mu \mathrm{m}$ ), polyacrylate (PA, $85 \mu \mathrm{m}$ ), polydimethylsiloxane (PDMS, $75 \mu \mathrm{m}$ ) and polydimethylsiloxane/divinylbenzene (PDMS/DVB, $65 \mu \mathrm{m}$ ), were used to evaluate the effect of fiber coating on the extraction efficiency of VOCs from wine vinegar samples. Figure S1 shows the typical total ion chromatograms (TIC) obtained using different fiber coatings with $5 \mathrm{~mL}$ of vinegar sample during $15 \mathrm{~min}$ at $40^{\circ} \mathrm{C}$ under constant magnetic stirring (700 rpm).

A total of 70 VOCs were identified using a PDMS fiber, while with the PDMS/DVB, CAR/PDMS, DVB/CAR/PDMS and PA fibers, were detected 68, 56, 63 and 63, respectively (Figure 2a). Each extraction was performed in triplicate and the precision (\% RSD) was lower than $15 \%$. According to the total GC peak area and obtained reproducibility, the most suitable fiber for the extraction of VOCs was the PDMS followed by the PDMS/DVB, CAR/PDMS, PA and DVB/CAR/PDMS. Therefore, the PDMS fiber was selected for further assays.

(a)

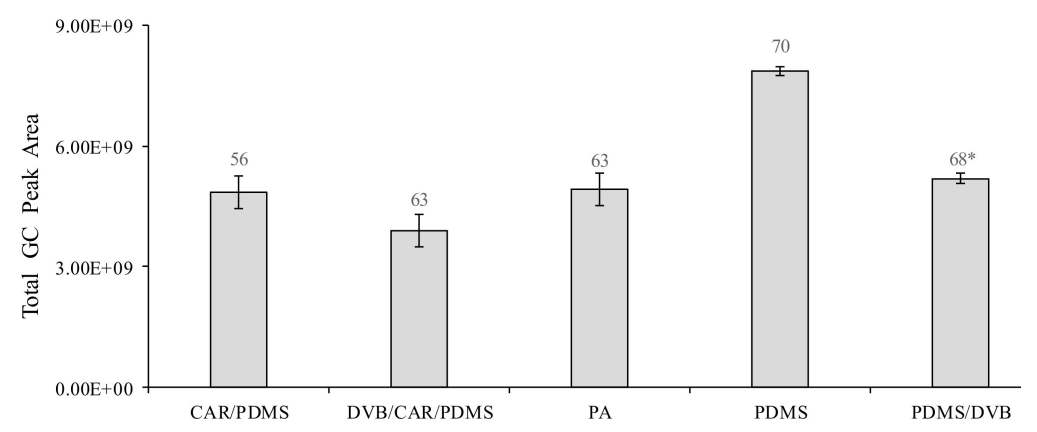

(b)

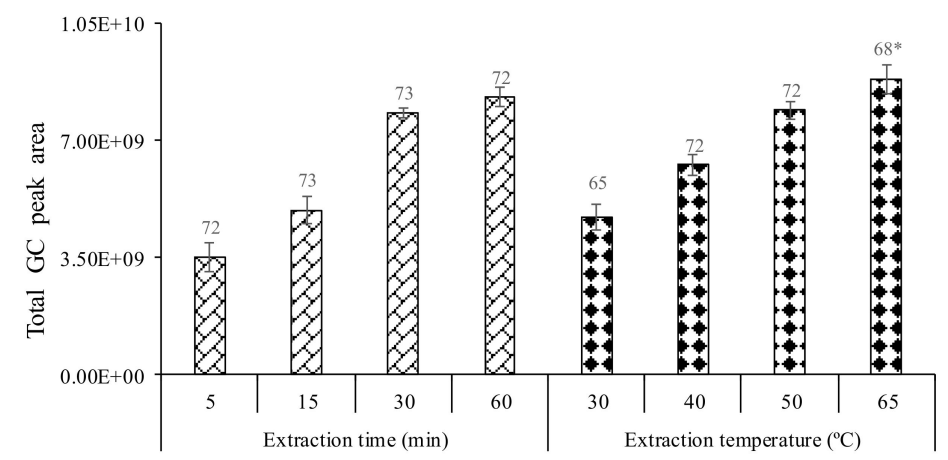

(c)

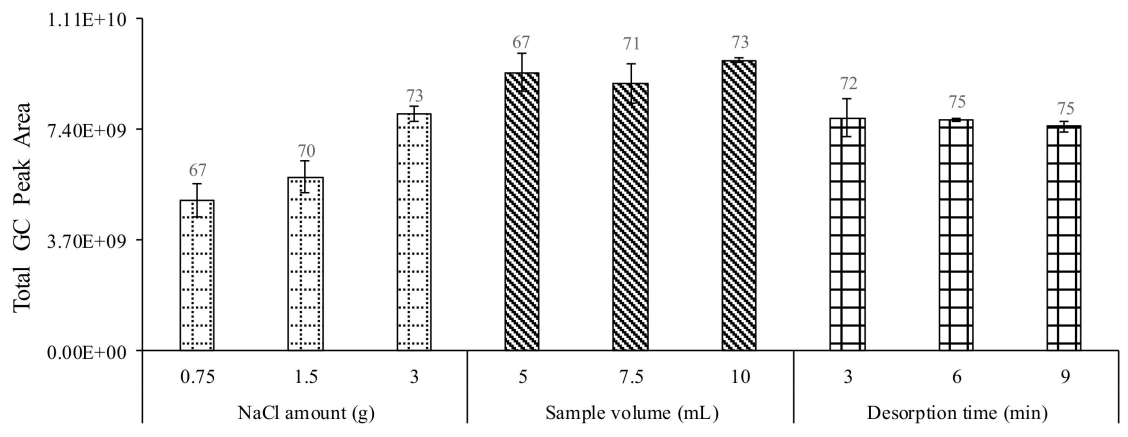

Figure 2. Effect of (a) fiber coating; (b) extraction time and extraction temperature; and (c) ionic strength, sample volume, desorption time on the volatile compounds extraction efficiency from wine vinegar. Error bars represent mean standard error ( $n=3$ for each data point). ${ }^{*}$ Number of identified compounds. 


\subsubsection{Extraction Time}

Time affects the mass transfer of the analytes between the three system phases in HS-SPME technique, mainly determined by the agitation rate and the partition coefficient of the analyte between fiber coating and sample matrix [28]. SPME has a maximum sensitivity at the equilibrium state, however full equilibration is not necessary for accurate and precise analysis. The extraction time for the PDMS fiber was assayed by plotting the GC response vs. the extraction time to obtain the partition equilibrium profile (Figure $2 b$ ). The HS-SPME extraction efficiency increase with extraction time. After 30 min no remarkable differences were observed in terms of GC response as well as number of identified VOCs. The highest $\%$ RSD values, were observed at 5 and $15 \mathrm{~min}$. These extraction times are too short to allow the system reach the equilibrium. Hence, an extraction time of $30 \mathrm{~min}$ was selected, since in terms of total GC peak areas, number of identified VOCs and precision, achieved the best performance.

\subsubsection{Extraction Temperature}

Temperature has a significant effect on the extraction kinetics by HS-SPME. An increase in extraction temperature causes an increase in extraction rate and a simultaneously decrease in the distribution constant. Therefore, an adequate temperature which provides suitable sensitivity and extraction rate should be used $[27,28]$. The extraction temperature effect was investigated by sampling wine vinegar at different temperatures, 30, 40, 50 and $65^{\circ} \mathrm{C}$ using the PDMS fiber, $5 \mathrm{~mL}$ of vinegar sample for 30 min under constant magnetic stirring $(800 \mathrm{rpm})$. As can be seen in Figure $2 \mathrm{~b}$, the best extraction efficiency was obtained at $65^{\circ} \mathrm{C}$ in terms of total GC peak area. In comparison with $50{ }^{\circ} \mathrm{C}$, the obtained results showed higher \% RSD whilst the number of VOCs identified was lower (68 VOCs). This decrease could be explained by VOCs degradation due to the higher temperature applied in the extraction procedure [29]. Taking into account the obtained results, $50{ }^{\circ} \mathrm{C}$ was selected as extraction temperature for further experiments.

\subsubsection{Ionic Strength}

The suitability of the HS-SPME for the extraction of VOCs depends on the transfer of the analyte from sample to the gaseous phase and therefore to the fiber. This process can be optimized by the increase of the ionic strength [28]. Different $\mathrm{NaCl}$ amounts $(0.75,1.5$ and $3 \mathrm{~g})$ were evaluated using a PDMS fiber with $5 \mathrm{~mL}$ of wine vinegar for $30 \mathrm{~min}$ at $50^{\circ} \mathrm{C}$ under constant magnetic stirring ( $800 \mathrm{rpm}$ ). As can be seen in Figure 2c, the total GC peak area as well as the number of identified VOCs increased as the $\mathrm{NaCl}$ amount increase, from 0.75 to $3 \mathrm{~g}$. Based on the obtained results, $3 \mathrm{~g}$ of NaCl was used to optimize the medium ionic strength and therefore promoting the "salting-out" effect, maximizing the extraction efficiency.

\subsubsection{Sample Volume}

The amount of sorbed analyte by the SPME fiber coating can be influenced by sample volume. It was expected, that the transition temperature would be lower if the headspace volume was higher. With a constant vial size, the sample volume should be reduced to increase the headspace volume, without affecting the extraction efficiency of SPME [30,31]. In order to evaluate the effect of sample volume on HS-SPME extraction efficiency, a sample volume ranging, from 5 to $10 \mathrm{~mL}$, was investigated using a PDMS fiber, $3 \mathrm{~g}$ of $\mathrm{NaCl}$ for $30 \mathrm{~min}$ at $50{ }^{\circ} \mathrm{C}$ under constant magnetic stirring ( $800 \mathrm{rpm}$ ). The sample volume of $10 \mathrm{~mL}$ was selected for the analysis since a total of 73 VOCs were identified, whilst by using 5 and $7.5 \mathrm{ml}$ of sample volume, were identified 66 and 71 VOCs, respectively (Figure 2c). In addition, the \% RSD for a volume of 5 and $7.5 \mathrm{~mL}$ of sample is high compared to $10 \mathrm{~mL}$. In terms of total GC peak area no remarkable differences were observed. Thus, $10 \mathrm{~mL}$ of sample was selected for the further assays. 


\subsubsection{Desorption Time}

Efficient thermal desorption of analytes in a GC injection port depends on the analytes volatility, the thickness of the fiber coating, injection depth, injection temperature and extraction time. Desorption time was evaluated in the range of 3 to $9 \mathrm{~min}$. As can be seen in Figure 2c, no remarkable differences in terms of GC peak area were observed for all tested desorption times using a PDMS fiber with $10 \mathrm{~mL}$ of vinegar sample and $3 \mathrm{~g}$ of $\mathrm{NaCl}$ for $30 \mathrm{~min}$ at $50{ }^{\circ} \mathrm{C}$ under constant magnetic stirring (800 rpm). Moreover, 75 VOCs were identified using 6 and 9 min of desorption time. Hence, 6 min was chosen for desorption of the VOCs from vinegar samples.

\subsection{Characterization of Volatile Signature of WBAV by Maceration Using HS-SPME/GC-MS}

Aroma is an important quality criteria for vinegars. Thus, the identification of VOCs responsible for its aroma is considered to be a key factor for quality and authentication control [4,19,22]. HS-SPME/GC-MS method was applied to establish the volatile signature of wine (control) and WBAV (banana, passion fruit, apple, pennyroyal). A total of 103 VOCs were identified in vinegar samples (Table 1) belonging to different chemical families, namely ethyl esters (37), alcohols (20), acids (10), terpenoids (23), carbonyl compounds (six), lactones (five) and volatile phenols (two). As far as we know, 34 of these VOCs are reported herein for the first time in vinegars (Table 1). These VOCs have been already identified by matching the obtained mass spectra with the reference compounds spectra in NIST Mass Spectral Search Program with a resemblance percentage above $80 \%$ and by comparison of the RIs calculated $\left(\mathrm{KI}_{\text {calc }}\right)$ with the values reported in the literature $\left(\mathrm{KI}_{\text {lit }}\right)$ for polyethylene glycol (or equivalent) column in vinegar samples using HS-SPME/GC-MS (Table 1). A range between 0 and $36\left(\left|\mathrm{KI}_{\text {calc }}-\mathrm{KI}_{\text {lit }}\right|\right)$ was obtained for $\mathrm{KI}_{\text {cal }}$ compared to the $\mathrm{KI}_{\text {lit }}$ reported in the literature for one dimensional GC with polyethylene glycol GC column or equivalent. This difference in KI is acceptable $(<5 \%)$ taking into account that the literature data is obtained from a large range of GC stationary phases (several commercial GC columns are composed of polyethylene glycol or equivalent stationary phases).

Forty-six VOCs were common in all vinegar samples analyzed, namely 17 ethyl esters, 11 alcohols, nine terpenoids, six acids, two lactones and one volatile phenol (Table 1). Moreover, some new VOCs, not found in wine vinegar (control) - mainly ethyl esters (e.g., ethyl 3-hexenoate) and terpenoids (e.g., limonene oxide, bornyl acetate, menthol)_appear in WBAV, as result of the maceration process. However, their contribution to the volatile signature was different for each vinegar sample, according to the raw material used. Table 1 shows the mean of GC peak area for each WBAV under study. The distribution of VOCs, according to its chemical family, is represented in Figure 3. Alcohols were the predominant chemical family in the studied vinegars. On average, the \% RPA of alcohols was higher in WBAV, ranging from 42.10 (banana vinegar) to $54.91 \%$ (apple vinegar), than in wine vinegar (control, 39.97\%). Ethanol, 3-methylbutanol and 2-phenylethanol were markedly the most abundant alcohols identified in all vinegar samples. The presence of these VOCs in vinegar samples can contribute positively with flower, fruity and sweet notes for sensory properties. Due to its importance in terms of quality and acceptance by consumers and because there are no published reports on this subject, the sensory expression of WBAV is being evaluated in an ongoing work aiming to define its main odor descriptors. In addition the limiar of olfactive perception of the most significant volatiles will be established in order to evaluate its contribution to the sensory characteristics of vinegars. 2-Hexanol, 2-heptanol, 1-octanol, 1-octne-3-ol, 2-nonanol, 2,3-butanediol isomer and methionol were detected in all WBAVs, with the exception on pennyroyal vinegar. Moreover, 2-butanol was not detected in WBAV macerated with passion fruit and apple. 
Table 1. Volatile organic compounds (VOCs) identified in wine-based aromatic vinegars (WBAVs) by headspace solid-phase microextraction (HS-SPME) PDMS/GC-MS.

\begin{tabular}{|c|c|c|c|c|c|c|c|c|c|c|c|c|c|c|}
\hline \multirow{3}{*}{$\begin{array}{c}\text { Peak } \\
\text { Number }\end{array}$} & \multirow{3}{*}{$\begin{array}{l}\mathrm{RT}^{1} \\
(\mathrm{~min})\end{array}$} & \multirow{3}{*}{$\mathrm{KI}_{\mathrm{cal}}{ }^{2}$} & \multirow{3}{*}{$\mathrm{KI}_{\text {lit }}{ }^{3}$} & \multirow{3}{*}{ Chemical Families } & \multicolumn{10}{|c|}{ GC Peak Area $\times 10^{6}(\mathrm{RSD})$} \\
\hline & & & & & \multirow{2}{*}{\multicolumn{2}{|c|}{$\begin{array}{l}\text { Wine Vinegar } \\
\text { Control }\end{array}$}} & \multicolumn{8}{|c|}{ Macerated Vinegars } \\
\hline & & & & & & & \multicolumn{2}{|c|}{ Banana } & \multicolumn{2}{|c|}{ Passion Fruit } & \multicolumn{2}{|c|}{ Apple } & \multicolumn{2}{|c|}{ Pennyroyal } \\
\hline & & & & & & & & & & & & & & \\
\hline 1 & 2.91 & 925 & 907 & Ethyl acetate & 300.22 & (8) & 197.65 & (9) & 122.23 & (13) & 113.89 & (9) & 247.35 & (3) \\
\hline 4 & 4.68 & 1055 & 1028 & Ethyl butanoate & 16.03 & (6) & -5 & & 9.93 & (14) & 3.29 & (4) & - & \\
\hline 5 & 5.29 & 1081 & 1050 & Ethyl 3-methylbutanoate & 15.18 & (6) & 16.36 & (17) & 3.31 & (13) & 8.12 & (4) & - & \\
\hline 7 & 6.51 & 1125 & 1120 & Isoamyl acetate & 163.77 & (15) & 169.1 & (9) & 71.31 & (3) & 80.64 & $(2)$ & 124.8 & (16) \\
\hline 12 & 10.04 & 1222 & 1220 & Ethyl hexanoate & 227.53 & (9) & 113.51 & (8) & 195.43 & (12) & 84.73 & (20) & 128.32 & (14) \\
\hline 17 & 12.67 & 1291 & 1292 & Ethyl 3-hexenoate ${ }^{4}$ & - & & 6.44 & (12) & 1.06 & (7) & 0.87 & (9) & 4.62 & (6) \\
\hline 18 & 13.23 & 1305 & 1300 & 3-Hexen-1-ol acetate isomer & 3.14 & $(4)$ & 0.94 & (15) & - & & 2.17 & (11) & - & \\
\hline 21 & 13.87 & 1320 & 1305 & Ethyl 2-hexenoate & 14.86 & (6) & 0.87 & (9) & 1.27 & (19) & 1.10 & $(4)$ & 2.81 & (5) \\
\hline 22 & 14.67 & 1339 & 1358 & Ethyl lactate & 21.95 & (14) & 42.24 & (16) & 36.08 & (4) & 28.80 & (14) & 51.13 & (1) \\
\hline 25 & 15.53 & 1343 & 1350 & Hexyl acetate & 22.48 & (17) & 29.76 & (11) & 18.26 & (9) & 16.00 & (2) & 12.48 & (13) \\
\hline 26 & 15.58 & 1359 & - & Heptyl acetate 4 & 0.52 & (15) & - & & - & & - & & - & \\
\hline 29 & 16.13 & 1370 & - & 2-Ethylhexyl acetate ${ }^{4}$ & 2.99 & (9) & 2.40 & (3) & - & & - & & - & \\
\hline 30 & 16.16 & 1372 & 1389 & Methyl octanoate & - & & 1.84 & (7) & 0.54 & (8) & - & & - & \\
\hline 34 & 17.82 & 1405 & 1394 & Ethyl 2-hydroxyisovalerate & - & & 2.46 & (11) & - & & 0.13 & (13) & - & \\
\hline 35 & 18.16 & 1416 & 1414 & Ethyl octanoate & 296.15 & (4) & 182.15 & (3) & 181.16 & (10) & 194.75 & (2) & 208.86 & (11) \\
\hline 47 & 22.33 & 1518 & 1483 & Ethyl 3-hydroxybutanoate & 2.65 & (13) & - & & 3.09 & (1) & 1.32 & (4) & - & \\
\hline 49 & 22.61 & 1526 & 1551 & 2-Ethyl hydroxycaproate ${ }^{4}$ & 6.45 & (19) & 8.08 & (6) & 2.62 & (14) & 0.42 & (11) & 6.02 & (8) \\
\hline 51 & 23.04 & 1536 & 1533 & Hexyl butanoate ${ }^{4}$ & 1.21 & $(7)$ & - & & - & & 1.74 & (3) & - & \\
\hline 64 & 26.58 & 1617 & 1636 & Ethyl decanoate & 112.62 & (3) & 132.17 & (14) & 110.55 & (5) & 211.67 & (4) & 138.31 & (2) \\
\hline 65 & 26.85 & 1625 & 1610 & Butyl octanoate ${ }^{4}$ & - & & 0.27 & (20) & - & & 1.47 & (16) & - & \\
\hline 66 & 27.35 & 1639 & 1648 & Ethyl benzoate & 6.63 & (15) & 6.26 & (8) & 5.83 & (11) & 6.65 & (1) & 9.15 & (4) \\
\hline 68 & 28.07 & 1659 & 1680 & Diethyl succinate & 25.99 & (5) & 23.95 & (15) & 15.49 & (4) & 18.84 & (2) & 23.72 & (5) \\
\hline 71 & 29.34 & 1693 & 1664 & Ethyl 3-hydroxyhexanoate & 1.27 & (4) & 1.02 & (9) & 0.20 & (12) & 0.21 & (20) & 0.98 & (5) \\
\hline 75 & 31.42 & 1754 & 1755 & Phenylmethyl acetate & 2.78 & (6) & - & & - & & 0.27 & (12) & - & \\
\hline 76 & 32.07 & 1773 & 1775 & Ethyl benzeneacetate & 11.12 & (14) & 7.44 & (8) & 4.61 & (8) & 3.95 & (7) & 11.51 & (7) \\
\hline 77 & 32.47 & 1784 & - & Dibuthyl succinate ${ }^{4}$ & 0.31 & (3) & - & & - & & 1.41 & (2) & - & \\
\hline 78 & 32.73 & 1791 & 1798 & Methyl 2-hydroxybenzoate & 0.63 & (17) & 6.07 & (10) & - & & - & & 4.23 & (2) \\
\hline 80 & 34.32 & 1838 & 1837 & Ethyl dodecanoate & 10.17 & (11) & 12.15 & (16) & 13.26 & (12) & 8.75 & (3) & 12.55 & (15) \\
\hline 82 & 34.99 & 1857 & 1821 & Benzyl propanoate 4 & 7.54 & (4) & 1.32 & (14) & 9.26 & (8) & 11.71 & (15) & 5.61 & (2) \\
\hline 83 & 35.58 & 1873 & 1849 & Benzyl butanoate 4 & 0.48 & (13) & - & & 1.37 & (11) & 0.58 & (16) & - & \\
\hline 84 & 35.87 & 1880 & 1883 & 2-Phenylethyl acetate & 1.00 & (11) & 5.73 & (5) & 1.19 & (8) & - & & 6.34 & (13) \\
\hline 85 & 36.47 & 1881 & 1880 & Citronellyl valerate 4 & 0.36 & (1) & 1.20 & (21) & 1.35 & (10) & 0.33 & (6) & 8.87 & (3) \\
\hline
\end{tabular}


Table 1. Cont

\begin{tabular}{|c|c|c|c|c|c|c|c|c|c|c|c|c|c|c|}
\hline \multirow{4}{*}{$\begin{array}{c}\text { Peak } \\
\text { Number } \\
90\end{array}$} & \multirow{4}{*}{$\begin{array}{l}\begin{array}{c}\mathrm{RT}^{1} \\
(\mathrm{~min})\end{array} \\
39.66\end{array}$} & \multirow{4}{*}{$\begin{array}{r}\mathbf{K I}_{\text {cal }}{ }^{2} \\
1970\end{array}$} & \multirow{4}{*}{$\begin{array}{r}\mathrm{KI}_{\text {lit }}{ }^{3} \\
1974\end{array}$} & \multirow{4}{*}{$\begin{array}{l}\text { Chemical Families } \\
\text { Methyl jasmonate } \\
\end{array}$} & \multicolumn{10}{|c|}{ GC Peak Area $\times 10^{6}($ RSD $)$} \\
\hline & & & & & \multirow{2}{*}{\multicolumn{2}{|c|}{$\begin{array}{c}\text { Wine Vinegar } \\
\text { Control }\end{array}$}} & \multicolumn{8}{|c|}{ Macerated Vinegars } \\
\hline & & & & & & & \multicolumn{2}{|c|}{ Banana } & \multicolumn{2}{|c|}{ Passion Fruit } & \multicolumn{2}{|c|}{ Apple } & \multicolumn{2}{|c|}{ Pennyroyal } \\
\hline & & & & & 0.98 & (2) & 0.81 & (4) & - & & 0.26 & (12) & - & \\
\hline 95 & 43.13 & 2193 & 2189 & Phenylethyl benzoate ${ }^{4}$ & 1.82 & (3) & 3.52 & (13) & 1.81 & (18) & 0.82 & $(4)$ & 2.20 & (11) \\
\hline \multirow[t]{2}{*}{100} & 48.08 & 2308 & 2301 & Methyl hexadecanoate & 0.41 & (6) & 1.99 & (12) & 0.45 & (6) & - & & 0.53 & $(14)$ \\
\hline & & & & Alcohols & & & & & & & & & & \\
\hline 2 & 3.15 & 968 & 972 & Ethanol & 773.10 & (10) & 845.14 & (11) & 1040.94 & (16) & 1099.28 & (12) & 946.25 & (6) \\
\hline 3 & 4.66 & 1074 & 1099 & 2-Butanol & 12.75 & (6) & 11.75 & (19) & - & & - & & 14.45 & (2) \\
\hline 6 & 6.12 & 1113 & 1112 & 2-Methyl-1-propanol & 23.13 & (12) & 13.42 & (9) & 9.14 & (12) & 14.55 & (17) & 19.72 & (8) \\
\hline 8 & 7.87 & 1165 & 1176 & 2-Hexanol & 2.75 & (7) & 3.25 & (10) & 1.86 & (12) & 2.19 & (15) & - & \\
\hline 11 & 9.51 & 1206 & 1206 & 3-Methylbutanol & 436.65 & (8) & 262.37 & (13) & 209.63 & (9) & 301.03 & (7) & 399.42 & (3) \\
\hline 20 & 13.51 & 1312 & 1332 & 2-Heptanol ${ }^{4}$ & 0.23 & (4) & 1.23 & (6) & 1.01 & (3) & 1.61 & (8) & - & \\
\hline 23 & 15.15 & 1350 & 1360 & 1-Hexanol & 23.03 & (10) & 33.89 & (13) & 69.49 & (3) & 55.64 & (10) & 63.41 & (3) \\
\hline 24 & 15.23 & 1352 & 1386 & 3-Hexenol isomer & 0.22 & (18) & 0.47 & (9) & 0.26 & (2) & 0.77 & (19) & 0.41 & (15) \\
\hline 27 & 15.81 & 1364 & 1379 & 3-Ethoxypropanol ${ }^{4}$ & 0.14 & (10) & 1.82 & (6) & 1.29 & (14) & - & & - & \\
\hline 28 & 16.11 & 1371 & 1391 & 3-Hexenol isomer & 5.78 & (3) & 1.83 & (16) & 2.01 & (11) & 8.56 & (13) & 6.49 & (4) \\
\hline 33 & 17.69 & 1383 & 1388 & 1-Octanol & 10.25 & (4) & 6.01 & (16) & 10.23 & (1) & 5.63 & (2) & - & \\
\hline 42 & 20.48 & 1475 & 1465 & 1-Octen-3-ol & 7.12 & (1) & 7.91 & (2) & 9.67 & (9) & 0.32 & (3) & - & \\
\hline 52 & 23.23 & 1540 & 1535 & 2-Nonanol ${ }^{4}$ & 0.53 & (8) & 1.91 & (3) & 4.37 & (7) & 3.13 & $(8)$ & - & \\
\hline 56 & 23.93 & 1556 & 1583 & 2,3-Butanediol isomer & 9.44 & (11) & 5.73 & (15) & 1.52 & (8) & 0.27 & (11) & - & \\
\hline 72 & 29.65 & 1701 & 1723 & Methionol & 1.21 & (2) & 1.39 & (19) & 1.35 & (5) & 1.66 & (8) & - & \\
\hline 73 & 31.28 & 1750 & 1765 & 1-Decanol ${ }^{4}$ & 1.60 & (12) & 5.82 & (12) & 1.08 & (17) & 2.22 & (17) & 8.32 & (9) \\
\hline 87 & 37.94 & 1898 & 1925 & 2-Phenylethanol & 124.77 & (5) & 78.46 & (12) & 27.58 & (7) & 63.08 & (6) & 68.75 & (6) \\
\hline \multirow[t]{2}{*}{89} & 39.24 & 1956 & 1952 & Tridecanol $^{4}$ & 0.67 & (7) & 2.14 & (12) & 6.68 & (10) & 0.99 & (12) & 4.35 & (2) \\
\hline & & & & Terpenoids & & & & & & & & & & \\
\hline 9 & 8.38 & 1178 & 1182 & Limonene & 101.05 & (1) & 82.77 & (6) & 79.66 & (12) & 78.23 & (3) & 104.46 & (8) \\
\hline 10 & 8.76 & 1187 & 1214 & Eucalyptol & 1.11 & $(4)$ & 4.21 & (21) & 2.13 & (2) & - & & 2.15 & (4) \\
\hline 13 & 11.18 & 1254 & 1233 & Sabinene & 2.22 & (15) & 6.03 & (2) & 1.19 & (15) & - & & - & \\
\hline 19 & 13.38 & 1308 & 1337 & Rose oxide isomer 4 & 8.25 & (3) & 8.31 & (2) & 9.78 & (12) & 9.23 & (1) & 9.77 & (4) \\
\hline 32 & 16.74 & 1392 & 1421 & Linalool oxide isomer & - & & - & & 1.45 & (12) & 0.33 & (10) & 8.76 & (6) \\
\hline 38 & 19.22 & 1443 & 1449 & Dihydrolinalool $^{4}$ & 1.32 & (8) & 9.83 & (14) & 1.37 & (20) & 2.61 & (12) & - & \\
\hline 39 & 19.4 & 1448 & 1467 & Linalool oxide isomer & - & & 3.32 & (14) & 0.96 & (7) & 0.18 & (6) & - & \\
\hline 40 & 19.97 & 1462 & 1474 & Menthone ${ }^{4}$ & - & & 8.42 & (9) & 4.19 & (2) & - & & 14.19 & (14) \\
\hline 41 & 20.16 & 1467 & 1467 & Limonene oxide & - & & 12.48 & (15) & 6.40 & (7) & 9.81 & (3) & 7.15 & (3) \\
\hline
\end{tabular}


Table 1. Cont

\begin{tabular}{|c|c|c|c|c|c|c|c|c|c|c|c|c|c|c|}
\hline \multirow{4}{*}{$\begin{array}{c}\begin{array}{c}\text { Peak } \\
\text { Number }\end{array} \\
44\end{array}$} & \multirow{4}{*}{$\begin{array}{l}\begin{array}{c}\mathrm{RT}^{\mathbf{1}} \\
(\mathrm{min})\end{array} \\
21.47\end{array}$} & \multirow{4}{*}{$\begin{array}{r}\mathrm{KI}_{\mathrm{cal}}{ }^{2} \\
1498\end{array}$} & \multirow{4}{*}{$\begin{array}{r}\mathrm{KI}_{\text {lit }}{ }^{3} \\
1531\end{array}$} & \multirow{4}{*}{$\begin{array}{c}\text { Chemical Families } \\
\text { Vitispirane I }\end{array}$} & \multicolumn{10}{|c|}{ GC Peak Area $\times 10^{6}($ RSD $)$} \\
\hline & & & & & \multirow{2}{*}{\multicolumn{2}{|c|}{$\begin{array}{l}\text { Wine Vinegar } \\
\text { Control }\end{array}$}} & \multicolumn{8}{|c|}{ Macerated Vinegars } \\
\hline & & & & & & & \multicolumn{2}{|c|}{ Banana } & \multicolumn{2}{|c|}{ Passion Fruit } & \multicolumn{2}{|c|}{ Apple } & \multicolumn{2}{|c|}{ Pennyroyal } \\
\hline & & & & & 5.61 & (8) & 6.21 & (15) & 3.95 & (1) & 4.95 & (5) & 6.57 & (5) \\
\hline 45 & 21.57 & 1501 & 1534 & Vitispirane II & 12.14 & (18) & 4.25 & (16) & 4.31 & (2) & 3.13 & (11) & 3.36 & (9) \\
\hline 50 & 22.88 & 1530 & 1537 & Linalool & 1.02 & $(8)$ & 3.35 & (7) & 6.15 & (4) & 2.15 & (4) & 1.98 & (4) \\
\hline 53 & 23.28 & 1541 & 1538 & Dihydrocarveol ${ }^{4}$ & - & & 11.61 & (3) & 14.14 & (5) & - & & 21.68 & $(14)$ \\
\hline 55 & 23.72 & 1551 & 1580 & Bornyl acetate ${ }^{4}$ & - & & 4.89 & (4) & 1.38 & (5) & 2.81 & $(8)$ & 1.18 & (15) \\
\hline 58 & 24.44 & 1559 & 1569 & Linalyl acetate & - & & 26.39 & (7) & 28.71 & (11) & - & & - & \\
\hline 59 & 24.83 & 1575 & 1574 & Fenchyl alcohol ${ }^{4}$ & 0.58 & (2) & 0.73 & (8) & 0.42 & (9) & 0.78 & (4) & 1.91 & (20) \\
\hline 62 & 26.27 & 1610 & 1626 & Menthol $^{4}$ & - & & 5.94 & (16) & 5.66 & (7) & 5.40 & (2) & 7.62 & (6) \\
\hline 63 & 26.34 & 1618 & 1632 & Pulegone ${ }^{4}$ & 2.84 & (13) & - & & 2.75 & (12) & - & & - & \\
\hline 69 & 28.58 & 1673 & 1669 & $\alpha$-Terpeniol & 49.62 & (12) & 60.22 & (9) & 64.55 & (9) & 65.23 & (2) & 67.71 & (3) \\
\hline 74 & 31.38 & 1753 & 1762 & Citronellol & 0.94 & (13) & 2.73 & $(4)$ & - & & - & & 1.72 & (12) \\
\hline 81 & 34.59 & 1844 & 1840 & Geranylacetone & 4.16 & (15) & 15.36 & (19) & 10.45 & (5) & 4.24 & (8) & 12.76 & (13) \\
\hline 91 & 39.86 & 1981 & 2009 & Nerolidol & 0.76 & $(8)$ & 0.82 & (3) & 1.21 & (4) & - & & 2.43 & (4) \\
\hline \multirow[t]{2}{*}{93} & 41.16 & 2125 & 2134 & $\alpha$-Cadinol & 4.23 & $(9)$ & 0.20 & (13) & 0.37 & $(2)$ & 1.09 & (7) & 2.06 & $(15)$ \\
\hline & & & & Carbonyl compounds & & & & & & & & & & \\
\hline 14 & 12.05 & 1276 & 1276 & Octanal & - & & - & & 0.52 & (7) & - & & - & \\
\hline 43 & 20.81 & 1483 & 1484 & Decanal & 1.69 & (8) & 2.19 & (13) & 1.90 & $(2)$ & 1.47 & (8) & - & \\
\hline 57 & 24.08 & 1571 & 1543 & Undecanone $^{4}$ & 2.72 & (12) & - & & 0.21 & (3) & - & & - & \\
\hline \multirow[t]{2}{*}{70} & 29.21 & 1689 & 1700 & Dodecanal $^{4}$ & 5.99 & (3) & - & & 0.94 & (6) & - & & - & \\
\hline & & & & Acids & & & & & & & & & & \\
\hline 36 & 18.51 & 1425 & 1407 & Acetic acid & 422.11 & (18) & 347.48 & (12) & 139.85 & (9) & 136.06 & (11) & 122.36 & (14) \\
\hline 54 & 23.52 & 1547 & 1572 & 2-Methylpropanoic acid & 1.52 & (14) & 2.66 & (7) & - & & - & & - & \\
\hline 61 & 25.97 & 1600 & 1619 & Butanoic acid & 3.77 & (1) & 8.09 & (15) & 0.92 & (4) & 0.44 & (9) & 1.25 & (7) \\
\hline 67 & 27.56 & 1645 & 1665 & Isovaleric acid & 19.86 & (13) & 9.15 & (12) & 2.61 & (7) & 6.63 & (11) & 16.27 & (5) \\
\hline 79 & 34.29 & 1837 & 1850 & Hexanoic acid & 37.85 & (11) & 32.83 & (4) & 17.25 & (8) & 15.06 & (5) & 21.49 & (10) \\
\hline 86 & 36.69 & 1937 & 1962 & 2-Hexenoic acid isomer ${ }^{4}$ & 0.23 & $(4)$ & 0.68 & (16) & 0.92 & (13) & - & & 1.68 & $(7)$ \\
\hline 94 & 41.82 & 2098 & 2083 & Octanoic acid & 83.66 & (7) & 41.12 & (13) & 34.35 & (3) & 53.22 & (2) & 76.83 & (12) \\
\hline 101 & 48.56 & 2321 & 2317 & Decanoic acid & 71.71 & (6) & 65.85 & (15) & 70.36 & (5) & 42.58 & (2) & 45.52 & (19) \\
\hline 102 & 54.81 & 2340 & 2310 & Hydrocinnamic acid & 10.63 & (10) & 1.02 & (16) & 0.81 & (7) & 2.43 & (5) & - & \\
\hline \multirow[t]{2}{*}{103} & 67.81 & 2392 & 2407 & Undecylic acid ${ }^{4}$ & 7.18 & $(8)$ & 0.91 & (15) & - & & 0.56 & $(8)$ & 0.62 & $(9)$ \\
\hline & & & & Lactones & & & & & & & & & & \\
\hline 60 & 25.71 & 1594 & 1618 & Butyrolactone & 0.94 & (5) & - & & - & & 0.19 & (19) & - & \\
\hline 88 & 37.95 & 1936 & 1912 & $\gamma$-Octalactone ${ }^{4}$ & 0.53 & (14) & - & & - & & - & & - & \\
\hline
\end{tabular}


Table 1. Cont.

\begin{tabular}{|c|c|c|c|c|c|c|c|c|c|c|c|c|c|c|}
\hline \multirow{4}{*}{$\begin{array}{c}\begin{array}{c}\text { Peak } \\
\text { Number }\end{array} \\
92\end{array}$} & \multirow{4}{*}{$\begin{array}{l}\begin{array}{c}\mathrm{RT}^{\mathbf{1}} \\
(\mathrm{min})\end{array} \\
40.63\end{array}$} & \multirow{4}{*}{$\begin{array}{c}\mathrm{KI}_{\text {cal }}{ }^{2} \\
2107\end{array}$} & \multirow{4}{*}{$\begin{array}{r}\mathbf{K I}_{\text {lit }}{ }^{3} \\
2103\end{array}$} & \multirow{4}{*}{$\begin{array}{c}\text { Chemical Families } \\
\gamma \text {-Decalactone }{ }^{4}\end{array}$} & \multicolumn{10}{|c|}{ GC Peak Area $\times 10^{6}($ RSD $)$} \\
\hline & & & & & \multirow{2}{*}{\multicolumn{2}{|c|}{$\begin{array}{l}\text { Wine Vinegar } \\
\text { Control }\end{array}$}} & \multicolumn{8}{|c|}{ Macerated Vinegars } \\
\hline & & & & & & & \multicolumn{2}{|c|}{ Banana } & \multicolumn{2}{|c|}{ Passion Fruit } & \multicolumn{2}{|c|}{ Apple } & \multicolumn{2}{|c|}{ Pennyroyal } \\
\hline & & & & & 2.28 & (17) & 0.95 & (8) & 2.28 & (2) & 14.59 & (2) & 4.23 & (4) \\
\hline 96 & 43.92 & 2218 & 2209 & Wine lactone ${ }^{4}$ & 3.99 & (2) & - & & - & & 1.27 & (7) & 2.60 & (12) \\
\hline \multirow[t]{2}{*}{99} & 45.44 & 2267 & 2241 & $\gamma$-Decenolactone ${ }^{4}$ & 11.22 & (6) & 4.43 & (8) & 7.34 & $(13)$ & 15.90 & (2) & 7.75 & $(1)$ \\
\hline & & & & Volatile phenols & & & & & & & & & & \\
\hline 97 & 44.22 & 2228 & 2205 & 4-Ethylphenol & 1.82 & (4) & 1.71 & (14) & 1.22 & (3) & - & & 0.79 & (3) \\
\hline
\end{tabular}

${ }^{4}$ Retention time $(\mathrm{min}) ;{ }^{2}$ Kovats index relative $n$-alkanes
Reported for the first time in vinegars; ${ }^{5}$ Not detected. 
Ethyl esters are qualitatively the main chemical family (37 VOCs), and represent the second major contribution to the WBAV volatile signature (from 28.18 (apple vinegar) to $35.48 \%$ (wine vinegar)). This chemical family contribute positively to the general quality of WBAV being responsible for their fruity and floral sensory properties. Among the ethyl esters, Table 1, ethyl hexanoate, ethyl octanoate, ethyl decanoate, isoamyl acetate and ethyl acetate are the most abundant, accounting $86.01 \%$ and $80.82 \%$ of total volatile profile of wine vinegar (control) and WBAV, respectively. Heptyl acetate $(0.04 \%)$ was only detected in wine-based vinegar (control), whereas, methyl octanoate (banana and passion fruit vinegars), ethyl 2-hydroxyisovalerate (banana and apple vinegars), isopentyl hexanoate (passion fruit and pennyroyal vinegars) and butyl octanoate (banana and apple vinegars) were detected only in WBAV. This fact suggest that these compounds come from raw material contributing to the flavor enrichment of macerated vinegars.

Fatty acids contributed with $18.26 \%$ and $10.95 \%$ for total volatile signature of wine vinegar and wine-based macerated vinegars, respectively. The lowest contribution was observed in pennyroyal $(8.89 \%)$, followed by apple $(8.99 \%)$, passion fruit $(9.44 \%)$, banana $(16.48 \%)$ and wine $(18.26 \%)$ vinegar. Acetic, octanoic and decanoic acids were predominant in the studied vinegar samples. Its contribution to the total volatile profile was highest in wine vinegar $(16.02 \%)$, followed by macerated wine vinegars, banana $(14.69 \%)$, passion fruit $(8.65 \%)$, apple $(8.11 \%)$ and pennyroyal $(7.60 \%)$.

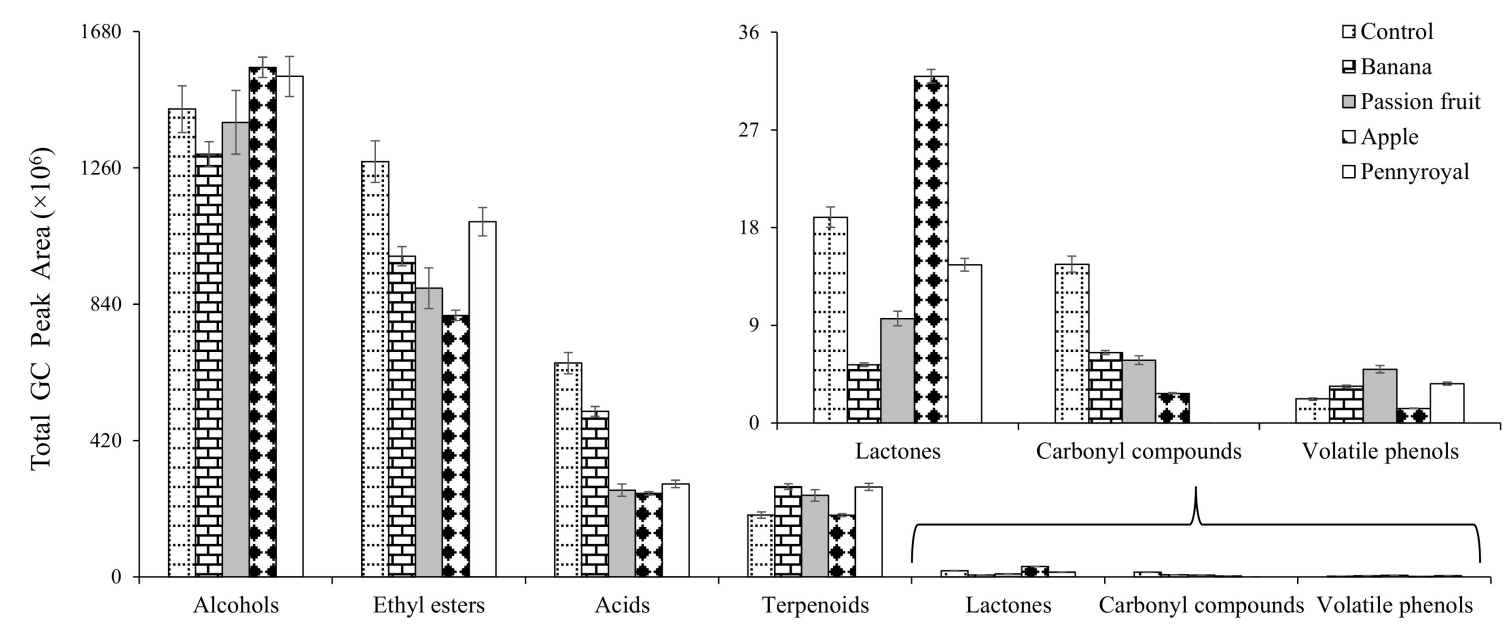

Figure 3. Total GC-MS peak area of chemical families identified in wine (control) and WBAV (banana, passion fruit, apple, pennyroyal) vinegars.

Terpenoids represent an excellent example of aromatic compounds from varietal origin, which may be used for authentication and/or typicality of food matrices. The WBAV macerated with banana seems to be richest in terpenoids $(8.99 \%)$, followed by passion fruit $(8.88 \%)$, pennyroyal $(8.62 \%)$, apple (6.65\%) and control (5.29\%). Moreover, linalool oxide isomer, menthone, limonene oxide, dihydrocarveol, bornyl acetate, linalyl acetate and menthol, were only detected in WBAVs (Table 1) suggesting the contribution of the maceration to the enrichment of wine vinegars. Limonene and $\alpha$-terpeniol were the predominant terpenoids accounting $78.95 \%, 51.42 \%, 57.41 \%, 75.44 \%$ and $62.05 \%$ for the total terpenoids in wine vinegar (control), and macerated vinegars with banana, passion fruit, apple and pennyroyal, respectively. From a healthy point of view, several studies demonstrated the effectiveness of limonene and $\alpha$-terpineol against some types of cancer including gastric, mammary, pulmonary adenoma and liver, as well as effective in relieving gastroesophageal reflux disorder and occasional heartburn $[34,35]$.

Carbonyl compounds were not detected in vinegars macerated with pennyroyal (Table 1), whereas octanal was only detected in vinegars macerated with passion fruit. Undecanone and dodecanal were only detected in wine vinegar (control) and vinegars macerated with passion fruit. This chemical 
group contribute with $0.41 \%$ for total volatile profile of wine vinegar (control), whereas in macerated vinegars its contribution ranged from 0.10 (apple) to $0.21 \%$ (banana).

In wine vinegar, the lactones contribute with $0.53 \%$ for the total volatile profile, whereas in vinegars macerated with banana, passion fruit, apple and pennyroyal, its contribution was $0.17 \%$, $0.34 \%, 1.12 \%$ and $0.45 \%$, respectively. $\gamma$-Octalactone was only detected in wine vinegar (control), whereas $\gamma$-decalactone and $\delta$-decenolactone were detected in all WBAV. From a sensorial point of view, lactones showed a positive contribution to aroma, with caramel, fruity, sweet and coconut-like notes [36].

\subsection{Multivariate Analysis}

The GC peak area of 103 VOCs (GC-MS data set) identified in wine vinegar (control) and WBAV were auto-scaled and submitted to PCA and HCA to search for the main sources of variability as well as to characterize and differentiate the vinegars based on their volatile signature.

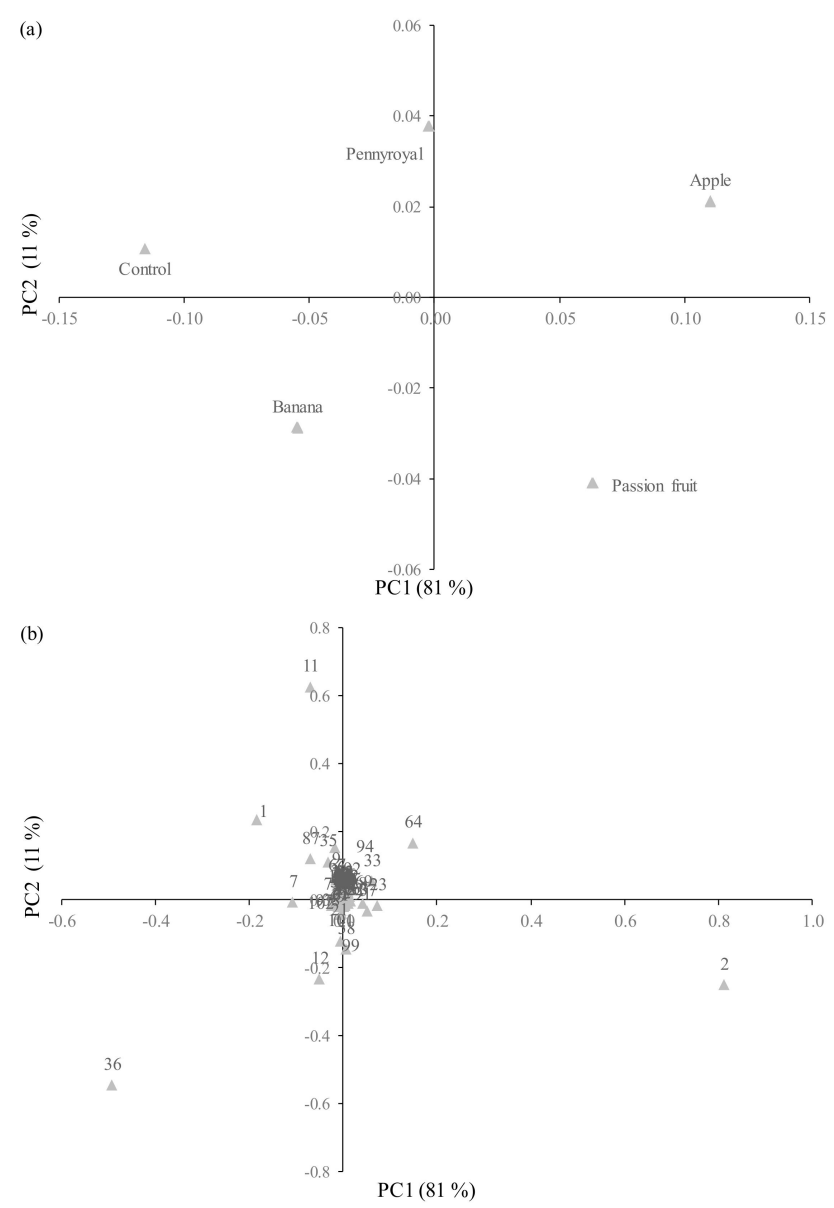

Figure 4. (a) Principal components (PC) PC1 $\times$ PC2 of scores scatter plot of wine (control) and WBAV vinegars; (b) Loading plot of the main source of variability between volatile profile and wine vinegars-derived products by maceration with fruits and plant (attribution of the peak number shown in Table 1).

PCA was used as the first exploratory step in our research to better understand the usefulness of the volatile signature to define and differentiate wine vinegar-derived products by maceration with fruits and medicinal herb. The PCA score scatter plot of the two first principal components, which explain $92 \%$ of total variability of GC-MS data, is represented in Figure $4 a$. The loadings of each VOCs (variables) that explain the differentiation among vinegar samples are shown in Figure $4 \mathrm{~b}$. 
According to results obtained by PCA, apple vinegar (PC1 and PC2 positive) is mainly characterized by the presence of ethyl decanoate (64), whereas banana vinegar (PC1 and PC2 negative) is essentially characterized by acetic acid (36). Wine vinegar (control) and pennyroyal vinegars were projected in PC1 negative and PC2 positive being essentially characterized by ethyl acetate (1) and 3-methylbutanol (11), respectively. The passion fruit vinegar (PC1 positive and PC2 negative) is described by ethanol (2).

The results of HCA for vinegar samples based on the GC-qMS data set is shown in Figure 5. The horizontal lines in the obtained dendrogram represent the similarity values between pairs of samples, between a sample and a group of samples and between sample groups, and the vertical lines the studied samples. The vinegar samples were firstly grouped into two clusters, i.e., cluster A (wine vinegar and banana vinegars) and cluster B (passion fruit, apple and pennyroyal vinegars). Moreover, cluster A could also be further grouped into sub-clusters, such as A1 (wine vinegar) and A2 (banana vinegar). These results are in agreement with those obtained by PCA, and it is possible to find that these clusters were evidently related to vinegar sample as well as to volatile signature.

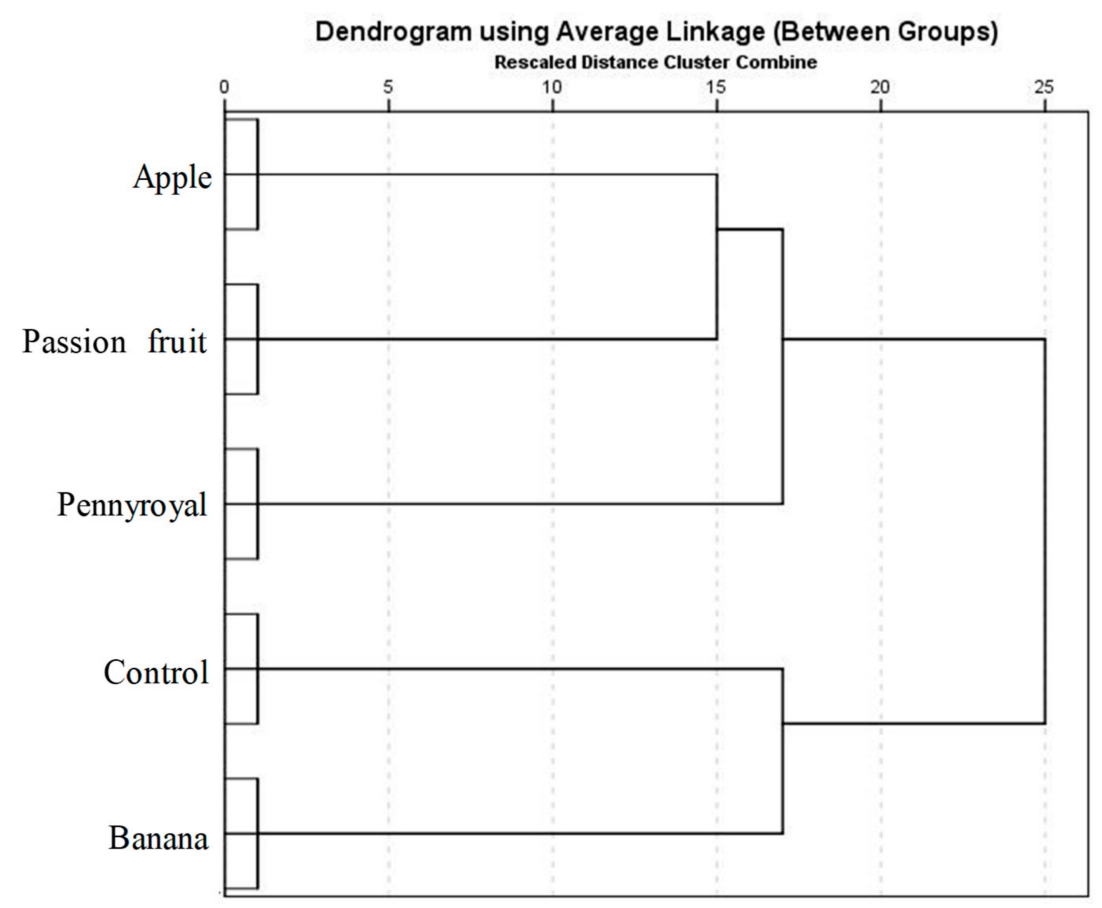

Figure 5. Dendrogram for the HCA results using Ward's cluster algorithm of the volatile profile obtained from wine (control) and WBAV vinegars. The Square Euclidean distances are shown on the $x$-axis.

\section{Materials and Methods}

\subsection{Chemicals and Materials}

All chemicals used in this study were of analytical quality. Sodium chloride $(\mathrm{NaCl}, 99.5 \%)$ used to adjust the ionic strength, was purchased from Panreac (Barcelona, Spain). VOCs standards used for identification of target compounds with purity $>98 \%$ were purchased from Sigma-Aldrich (Madrid, Spain), Acros Organics (Geel, Belgium) and Fluka (Buchs, Switzerland). The individual stock solutions were prepared in ethanol (concentration of $1000 \mathrm{mg} / \mathrm{L}$ ) and stored at $4{ }^{\circ} \mathrm{C}$. Helium (Air Liquide, Miraflores, Algés, Portugal) was used as GC carrier gas. The glass vials, SPME fibers, and SPME holder for manual sampling were purchased from Supelco (Bellefonte, PA, USA). The Kovats index (KI) was calculated through injection of a series of $C_{8}$ to $C_{20}$ straight-chain $n$-alkanes (concentration of $40 \mathrm{mg} / \mathrm{L}$ in $n$-hexane) was supplied by Fluka. 


\subsection{Vinegar Samples}

The WBAVs were obtained according to Procedure I in Figure 1. For this purpose, individual maceration of wine vinegar with different fruits and the medicinal herb was carried out. The vinegar used to perform the maceration was supplied by a local winery. The fruits used for maceration, banana (Musa sapientum L.), passion fruit (Passiflora edulis L.), apple (Malus domestica Borkh.) and the medicinal herb (pennyroyal (Mentha pulegium L.)), were purchased from a local market at optimal maturity and health stage. Samples were washed and dried to remove possible contaminants.

To promote the enrichment of volatile profile through maceration process, dark glass containers $(1000 \mathrm{~mL})$ were filled with $750 \mathrm{~mL}$ of wine vinegar, to which small pieces $(50 \mathrm{~g})$ of banana, passion fruit, apple and pennyroyal were added. All dark glass containers were continuously stirred during 7 days at room temperature $\left(25 \pm 1^{\circ} \mathrm{C}\right)$. The wine vinegar without maceration was used as control vinegar. Each assay was performed in triplicate.

\subsection{HS-SPME Procedure}

To improve the extraction efficiency of HS-SPME several parameters such as: fiber coating, extraction time $(5,15,30,60 \mathrm{~min})$, extraction temperature $\left(30,40,50,60^{\circ} \mathrm{C}\right)$, sample volume $(5,7.5$, $10 \mathrm{~mL})$, ionic strength $(0.75,1.5,3 \mathrm{~g})$ and desorption time $(3,6,9 \mathrm{~min})$ were tested and optimized using an univariate experimental design.

For fiber screening, five commercial fibers, namely carboxen/polydimethylsiloxane (CAR/PDMS, $75 \mu \mathrm{m}$ ), divinylbenzene/carboxen/polydimethylsiloxane (DVB/CAR/PDMS, 50/30 $\mu \mathrm{m}$ ), polyacrylate (PA, $85 \mu \mathrm{m}$ ), polydimethysiloxane (PDMS, $100 \mu \mathrm{m}$ ) and polydimethylsiloxane/divinylbenzene (PDMS/DVB, $65 \mu \mathrm{m}$ ) were evaluated. Before daily analyses each fiber was conditioned for $15 \mathrm{~min}$ at $250{ }^{\circ} \mathrm{C}$.

For HS-SPME optimization, a wine-based vinegar (Control) was used. For each extraction, $10 \mathrm{~mL}$ of sample was transferred to a $20 \mathrm{~mL}$ amber glass vial with $3 \mathrm{~g}$ of $\mathrm{NaCl}$. A magnetic stirrer bar was added. The vial, tightly capped with a PTFE-faced silicone septum, was placed in a thermostatic block with a constant magnetic stirring $(800 \mathrm{rpm})$. The SPME fiber was exposed into the headspace during $30 \mathrm{~min}$ at $50^{\circ} \mathrm{C}$. Subsequently, after extraction the fiber was withdrawn into the holder needle, removed from the vial and immediately introduced into the GC injector port for 6 min at $250{ }^{\circ} \mathrm{C}$ for thermal desorption of the analytes. All assays were performed in triplicate.

\subsection{GC-MS Conditions}

An Agilent 6890N gas chromatograph system (Palo Alto, CA, USA) combined with an Agilent 5975 quadrupole inert mass selective detector and a splitless injector was used. Chromatographic separations were performed using a BP-20 (30 $\mathrm{m} \times 0.25 \mathrm{~mm}$ i.d. $\times 0.25 \mu \mathrm{m}$ film thickness) fused silica capillary column supplied by SGE (Darmstadt, Germany) with helium (Helium N60, Air Liquide) as carrier gas at a flow rate of $1 \mathrm{~mL} / \mathrm{min}$ (column-head pressure: $13 \mathrm{psi}$ ). An insert of $0.75 \mathrm{~mm}$ i.d. was used and the injector temperature was fixed to $250^{\circ} \mathrm{C}$. The temperature program was programmed as follows: initial temperature $40{ }^{\circ} \mathrm{C}$ and a ramp of $3{ }^{\circ} \mathrm{C} \mathrm{min}-1$ to $220^{\circ} \mathrm{C}$ and maintaining a constant temperature for $10 \mathrm{~min}$ until the end. The manifold, GC-MS interface and quadrupole temperatures were held at 180,220 and $180^{\circ} \mathrm{C}$, respectively. MS detection was performed in full scan, the ion energy used for the electron impact (EI) was $70 \mathrm{eV}$ and the source temperature was $180^{\circ} \mathrm{C}$. The electron multiplier was set to the auto tune procedure. The mass acquisition range, made in full scan mode, was $30-300 \mathrm{~m} / \mathrm{z}, 1.9$ spectra $\mathrm{s}^{-1}$. VOCs identification was achieved by the following ways:

(i) comparison the GC retention times and mass spectra with those of the standard, when available;

(ii) all mass spectra were also compared with the data system library (NIST Mass Spectral Search Program v.2.0d software; NIST: Washington, DC, USA, 2005);

(iii) Kovats index (KI) values were determined according to the van den Dool and Kratz equation [37]. 
For the determination of the $\mathrm{KI}, \mathrm{a} \mathrm{C}_{8}-\mathrm{C}_{20} n$-alkanes series was used, and the values were compared, when available, with values reported in the literature for similar columns $[5,27,32,33]$.

\subsection{Statistical Analysis}

Chemometric tools, namely PCA and HCA, were applied to the auto-scaled areas of the identified VOCs in wine-based aromatic vinegars (WBAV), using SPSS version 23 Statistical Package for Windows (SPSS Inc., Chicago, IL, USA). Auto-scaling is a data pre-treatment process that makes variables of different scales comparable. Each variable is autoscaled separately by subtracting its mean value and dividing by its standard deviation. The HCA was processed employing Ward's minimum variance algorithm method, and the distance was determined using squared Euclidean distances. The Ward's algorithm consists in aggregating two clusters such that the growth of within-inertia is minimum, i.e., minimizing the reduction of the between-inertia, at each step of the algorithm. The within inertia characterizes the homogeneous of a cluster. The goal was to extract the main sources of variability and hence to help on the characterization of the dataset [38].

\section{Conclusions}

The optimized method based on the HS-SPME/GC-MS was revealed as a suitable tool to establish the volatile signature of WBAVs obtained by maceration with fruits and a medicinal herb. A total of 103 VOCs, belonging to different chemical families, were identified. In terms of $\%$ RPA values and VOCs identified differences were observed between wine vinegar and WBAV, as well as among WBAV. In the case of WBAV, the different contribution for total volatile profile could be explained by the fruit volatile signature used to macerate the wine vinegar (control). The fruit volatile signature can be affected by climatic conditions, sun exposition and agronomic practices. Higher alcohols, ethyl esters and fatty acids were the predominant chemical families found in all investigated vinegar samples. On the other hand, in qualitative terms, carbonyl compounds were not detected in pennyroyal vinegar, whereas ethyl 3-ethoxypropanoate, ethyl 3-hexenoate, methyl octanoate, ethyl 2-hydroxyisovalerate, isopentyl hexanoate, butyl octanoate, linalool oxide isomer, menthone, limonene oxide, dihydrocarveol, bornyl acetate, linalyl acetate, menthol and octanal were only detected in some WBAVs. From a sensorial point of view, the ethyl esters and terpenoids only detected in some WBAV showed a positive contribution to aroma with fruity, sweet, green, fresh, anise and berry notes to WBAV aroma, since they have low odor threshold (in order of $\mu \mathrm{g} / \mathrm{L}$ ). However, a future work will be done related to sensorial analysis of WBAV using HS-SPME/GC-O in order to define its odor descriptors and provide their distinct patterns based on their volatile signature depending on the raw material used in the maceration process. As far as we known, no studies are available related to sensorial analysis of WBAV and therefore it will be subject of a future publication.

The HS-SPME/GC-MS data combined with PCA and HCA provided a powerful tool to differentiate vinegar from WBAV based on volatile signature. This knowledge represents a suitable tool to guarantee the authenticity and genuineness of wine as well wine-based aromatic vinegars, to promote the production of vinegars with enhanced levels of volatile compounds. As far as we know, 34 VOCs were identified for the first time in vinegars.

Supplementary Materials: Supplementary materials are available online.

Acknowledgments: The authors acknowledge the FCT-Fundação para a Ciência e a Tecnologia through the pos-Doc grant (SFRH/BPD/97387/2013), PhD grant (SFRH/BD/97039/2013), MS Portuguese Networks (REDE/1508/RNEM/2010) and CQM grant, PEst-UID/QUI/00674/2013 (CQM is a FCT-National Research Unit). Funding through the project M1420-01-0145-FEDER-000005-Centro de Química da Madeira-CQM+(Madeira 14-20), is also acknowledged.

Author Contributions: R.P. performed the design of experiments, optimization experimental parameters, and data analysis; C.L.S. and P.S. performed the support to the experiments, GC-MS and data analysis. J.S.C. performed the conception of study, and manuscript preparation and editing.

Conflicts of Interest: The authors declare no conflict of interest. 


\section{References}

1. Ubeda, C.; Callejón, R.M.; Hidalgo, C.; Torija, M.J.; Mas, A.; Troncoso, A.M.; Morales, M.L. Determination of major volatile compounds during the production of fruit vinegars by static headspace gas chromatography-mass spectrometry method. Food Res. Int. 2011, 44, 259-268. [CrossRef]

2. Budak, N.; Aykin, .; Seydim, A.; Greene, A.K.; Guzel-Seydim, Z.B. Functional properties of vinegar. J. Food Sci. 2014, 79, R757-R764. [CrossRef] [PubMed]

3. Budak, H.; Guzel-Seydim, Z. Antioxidant activity and phenolic content of wine vinegars produced by two different techniques. J. Sci. Food Agric. 2010, 90, 2021-2026. [CrossRef] [PubMed]

4. Jo, D.; Kim, G.-R.; Yeo, S.-H.; Jeong, Y.-J.; Noh, B.S.; Kwon, J.-H. Analysis of aroma compounds of commercial cider vinegars with different acidities using SPME/GC-MS, electronic nose, and sensory evaluation. Food Sci. Biotechnol. 2013, 22, 1559-1565. [CrossRef]

5. Cejudo-Bastante, M.J.; Rodríguez Dodero, M.C.; Durán Guerrero, E.; Castro Mejías, R.; Natera Marín, R.; García Barroso, C. Development and optimisation by means of sensory analysis of new beverages based on different fruit juices and sherry wine vinegar. J. Sci. Food Agric. 2013, 93, 741-748. [CrossRef] [PubMed]

6. Cejudo-Bastante, C.; Castro-Mejías, R.; Natera-Marín, R.; García-Barroso, C.; Durán-Guerrero, E. Chemical and sensory characteristics of orange based vinegar. J. Food Sci. Technol. 2016, 53, 3147-3156. [CrossRef] [PubMed]

7. Chen, H.; Chen, T.; Giudici, P.; Chen, F. Vinegar Functions on Health: Constituents, Sources, and Formation Mechanisms. Compr. Rev. Food Sci. Food Saf. 2016, 15, 1124-1138. [CrossRef]

8. Marrufo-Curtido, A.; Cejudo-Bastante, M.J.; Rodríguez-Dodero, M.C.; Natera-Marín, R.; Castro-Mejías, R.; García-Barroso, C.; Durán-Guerrero, E. Novel vinegar-derived product enriched with dietary fiber: Effect on polyphenolic profile, volatile composition and sensory analysis. J. Food Sci. Technol. 2015, 52, 7608-7624. [CrossRef] [PubMed]

9. Venturi, F.; Sanmartin, C.; Taglieri, I.; Nari, A.; Andrich, G.; Terzuoli, E.; Donnini, S.; Nicolella, C.; Zinnai, A. Development of phenol-enriched olive oil with phenolic compounds extracted from wastewater produced by physical refining. Nutrients 2017, 9, 916. [CrossRef] [PubMed]

10. Lorenzen, J.; Igl, N.; Tippelt, M.; Stege, A.; Qoura, F.; Sohling, U.; Brück, T. Extraction of microalgae derived lipids with supercritical carbon dioxide in an industrial relevant pilot plant. Bioprocess Biosyst. Eng. 2017, 40, 911-918. [CrossRef] [PubMed]

11. Zinnai, A.; Sanmartin, C.; Taglieri, I.; Andrich, G.; Venturi, F. Supercritical fluid extraction from microalgae with high content of LC-PUFAs. A case of study: $\mathrm{Sc}-\mathrm{CO}_{2}$ oil extraction from Schizochytrium sp. J. Supercrit. Fluids 2016, 116, 126-131. [CrossRef]

12. Ghitescu, R.-E.; Volf, I.; Carausu, C.; Bühlmann, A.-M.; Gilca, I.A.; Popa, V.I. Optimization of ultrasound-assisted extraction of polyphenols from spruce wood bark. Ultrason. Sonochem. 2015, 22, 535-541. [CrossRef] [PubMed]

13. Wang, Y.-Z.; Fu, S.-G.; Wang, S.-Y.; Yang, D.-J.; Wu, Y.-H. S.; Chen, Y.-C. Effects of a natural antioxidant, polyphenol-rich rosemary (Rosmarinus officinalis L.) extract, on lipid stability of plant-derived omega-3 fatty-acid rich oil. LWT 2018, 89, 210-216. [CrossRef]

14. Chinnici, F.; Durán Guerrero, E.; Sonni, F.; Natali, N.; Natera Marín, R.; Riponi, C. Gas Chromatography-Mass Spectrometry (GC-MS) Characterization of Volatile Compounds in Quality Vinegars with Protected European Geographical Indication. J. Agric. Food Chem. 2009, 57, 4784-4792. [CrossRef] [PubMed]

15. Yu, Y.-J.; Lu, Z.-M.; Yu, N.-H.; Xu, W.; Li, G.-Q.; Shi, J.-S.; Xu, Z.-H. HS-SPME/GC-MS and chemometrics for volatile composition of Chinese traditional aromatic vinegar in the Zhenjiang region. J. Inst. Brew. 2012, 118, 133-141. [CrossRef]

16. Shu, X.; Jiang, X.-W.; Cheng, B.C.-Y.; Ma, S.-C.; Chen, G.-Y.; Yu, Z.-L. Ultra-performance liquid chromatography-quadrupole/time-of-flight mass spectrometry analysis of the impact of processing on toxic components of Kansui Radix. BMC Complement. Altern. Med. 2016, 16, 73. [CrossRef] [PubMed]

17. Pinu, F.; de Carvalho-Silva, S.; Trovatti Uetanabaro, A.; Villas-Boas, S. Vinegar Metabolomics: An Explorative Study of Commercial Balsamic Vinegars Using Gas Chromatography-Mass Spectrometry. Metabolites 2016, 6, 22. [CrossRef] [PubMed] 
18. Roberto, R.M.; García, N.P.; Hevia, A.G.; Valles, B.S. Application of purge and trap extraction and gas chromatography for determination of minor esters in cider. J. Chromatogr. A 2005, 1069, 245-251. [CrossRef]

19. Guerrero, E.D.; Marín, R.N.; Mejías, R.C.; Barroso, C.G. Stir bar sorptive extraction of volatile compounds in vinegar: Validation study and comparison with solid phase microextraction. J. Chromatogr. A 2007, 1167, 18-26. [CrossRef] [PubMed]

20. Xing, J.; Sun, H.-M.; Li, Z.-Y.; Qin, X.-M. Comparison of volatile components between raw and vinegar baked radix bupleuri by GC-MS based metabolic fingerprinting approach. Evid.-Based Complement. Alternat. Med. eCAM 2015, 2015, 653791. [CrossRef] [PubMed]

21. Fernández de Simón, B.; Martínez, J.; Sanz, M.; Cadahía, E.; Esteruelas, E.; Muñoz, A.M. Volatile compounds and sensorial characterisation of red wine aged in cherry, chestnut, false acacia, ash and oak wood barrels. Food Chem. 2014, 147, 346-356. [CrossRef] [PubMed]

22. Barnaba, C.; Dellacassa, E.; Nicolini, G.; Nardin, T.; Malacarne, M.; Larcher, R. Identification and quantification of 56 targeted phenols in wines, spirits, and vinegars by online solid-phase extraction-Ultrahigh-performance liquid chromatography-Quadrupole-orbitrap mass spectrometry. J. Chromatogr. A 2015, 1423, 124-135. [CrossRef] [PubMed]

23. Xiao, Z.; Dai, S.; Niu, Y.; Yu, H.; Zhu, J.; Tian, H.; Gu, Y. Discrimination of chinese vinegars based on headspace solid-phase microextraction-gas chromatography mass spectrometry of volatile compounds and multivariate analysis. J. Food Sci. 2011, 76, C1125-C1135. [CrossRef] [PubMed]

24. Durán Guerrero, E.; Chinnici, F.; Natali, N.; Marín, R.N.; Riponi, C. Solid-phase extraction method for determination of volatile compounds in traditional balsamic vinegar. J. Sep. Sci. 2008, 31, 3030-3036. [CrossRef] [PubMed]

25. Pereira, A.C.; Reis, M.S.; Saraiva, P.M.; Marques, J.C. Analysis and assessment of Madeira wine ageing over an extended time period through GC-MS and chemometric analysis. Anal. Chim. Acta 2010, 660, 8-21. [CrossRef] [PubMed]

26. Perestrelo, R.; Barros, A.S.; Rocha, S.M.; Câmara, J.S. Establishment of the varietal profile of Vitis vinifera L. grape varieties from different geographical regions based on HS-SPME/GC-qMS combined with chemometric tools. Microchem. J. 2014, 116, 107-117. [CrossRef]

27. Spínola, V.; Perestrelo, R.; Câmara, J.S.; Castilho, P.C. Establishment of Monstera deliciosa fruit volatile metabolomic profile at different ripening stages using solid-phase microextraction combined with gas chromatography-mass spectrometry. Food Res. Int. 2015, 67, 409-417. [CrossRef]

28. Ferreira, L.; Perestrelo, R.; Câmara, J.S. Comparative analysis of the volatile fraction from Annona cherimola Mill. cultivars by solid-phase microextraction and gas chromatography-quadrupole mass spectrometry detection. Talanta 2009, 77, 1087-1096. [CrossRef] [PubMed]

29. Sánchez-Palomo, E.; Díaz-Maroto, M.C.; Pérez-Coello, M.S. Rapid determination of volatile compounds in grapes by HS-SPME coupled with GC-MS. Talanta 2005, 66, 1152-1157. [CrossRef] [PubMed]

30. Ferreira, L.; Perestrelo, R.; Caldeira, M.; Câmara, J.S. Characterization of volatile substances in apples from Rosaceae family by headspace solid-phase microextraction followed by GC-qMS. J. Sep. Sci. 2009, 32, 1875-1888. [CrossRef] [PubMed]

31. Zhang, C.; Qi, M.; Shao, Q.; Zhou, S.; Fu, R. Analysis of the volatile compounds in Ligusticum chuanxiong Hort. using HS-SPME-GC-MS. J. Pharm. Biomed. Anal. 2007, 44, 464-470. [CrossRef] [PubMed]

32. Varming, C.; Petersen, M.A.; Poll, L. Comparison of isolation methods for the determination of important aroma compounds in black currant (Ribes nigrum L.) juice, using nasal impact frequency profiling. J. Agric. Food Chem. 2004, 52, 1647-1652. [CrossRef] [PubMed]

33. Lee, S.-J.; Noble, A.C. Characterization of odor-active compounds in Californian chardonnay wines using GC-olfactometry and GC-mass spectrometry. J. Agric. Food Chem. 2003, 51, 8036-8044. [CrossRef] [PubMed]

34. Sun, J. D-Limonene: Safety and Clinical Applications. Alternat. Med. Rev. A J. Clin. Ther. 2007, 1212, $259-264$.

35. Gautam, N.; Mantha, A.K.; Mittal, S. Essential oils and their constituents as anticancer agents: A mechanistic view. BioMed Res. Int. 2014, 2014, 154106. [CrossRef] [PubMed]

36. El Hadi, M.; Zhang, F.-J.; Wu, F.-F.; Zhou, C.-H.; Tao, J. Advances in fruit aroma volatile research. Molecules 2013, 18, 8200-8229. [CrossRef] [PubMed] 
37. Van Den Dool, H.; Dec. Kratz, P. A generalization of the retention index system including linear temperature programmed gas_Liquid partition chromatography. J. Chromatogr. A 1963, 11, 463-471. [CrossRef]

38. Vidal, R.; Ma, Y.; Sastry, S.S. Generalized Principal Component Analysis; Springer: New York, NY, USA, 2016; pp. 25-62. ISBN 978-0-387-87811-9.

Sample Availability: Samples of the compounds are available from the authors.

(C) 2018 by the authors. Licensee MDPI, Basel, Switzerland. This article is an open access article distributed under the terms and conditions of the Creative Commons Attribution (CC BY) license (http:/ / creativecommons.org/licenses/by/4.0/). 